\title{
UM ESTUDO SOBRE A INCIDÊNCIA DE DENGUE EM MUNICÍPIOS DA REGIÃO DA ALTA PAULISTA
}

\section{A study on the incidence of dengue in counties in the region of Alta Paulista}

Rayane Arissa Aikawa, Sandra Cristina Oliveira

Universidade Estadual Paulista - UNESP, Tupã, SP.

E-mail: rayane.aikawa@unesp.br , sandra@tupa.unesp.br

RESUMO - Atualmente a dengue é considerada um dos maiores problemas de saúde pública no Brasil e no mundo, especialmente em regiões tropicais, onde as condições ambientais (temperatura, precipitação pluviométrica, processo de urbanização, etc.) favorecem a presença de criadouros, que permitem o desenvolvimento e a proliferação do mosquito Aedes Aegypti, o vetor da doença. $\mathrm{O}$ aumento do número de casos de dengue é resultado de vários fatores, que facilitam a introdução, a transmissão e a dispersão do vírus, tais como: a utilização de meios de transportes cada vez mais rápidos; o aumento do processo de migração humana; a urbanização desordenada; a fragilidade da estrutura e planejamento, principalmente das periferias; o acréscimo do uso de recipientes descartáveis e a falta de saneamento ambiental. Assim, o objetivo deste trabalho foi analisar a incidência de dengue em municípios da região da Alta Paulista. Especificamente, foram ajustados modelos fixos de séries temporais para séries históricas de incidência de dengue nos seis principais municípios da referida região e foram obtidas previsões de incidência, usando as modelagens que proporcionaram melhores ajustes aos dados. Os resultados mostraram que os modelos de alisamento exponencial são os mais robustos para ajustar os dados de incidência de dengue e, consequentemente para realizar previsões, permitindo que as ações de prevenção e controle da dengue sejam contínuas.

Palavras-chave: Aedes aegypti; Alisamento exponencial; Previsão; Gestão pública.

ABSTRACT - Currently, dengue is considered one of the biggest public health problems in Brazil and in the world, especially in tropical regions, where environmental conditions (temperature, rainfall, the urbanization process, etc.) favor the presence of breeding sites, which allow the development and the proliferation of the Aedes Aegypti mosquito, the vector of the disease. The increase in the number of dengue cases is the result of several factors, which facilitate the introduction, transmission, and spread of the virus, such as the use of increasingly faster means of transport; the increase in the human migration process; the disorderly urbanization; the fragility of the structure and planning, mainly of the peripheries; the increase in the use of disposable containers and the lack of environmental sanitation. Thus, the general aim of this work was to analyze the incidence of dengue in counties in the region of Alta Paulista. Specifically, fixed time series models were adjusted for historical 
dengue incidence series in the six main counties in that region and incidence forecasts were obtained, using the models that provided better adjustments to the data. The results showed that the exponential smoothing models are the most robust to adjust the dengue incidence data and, consequently to forecast, allowing dengue prevention and control actions to be continuous.

Keywords: Aedes aegypti; Exponential smoothing; Forecasting; Public administration

\section{INTRODUÇÃO}

A dengue é uma doença considerada arbovirose, já que é causada por um arbovírus pertencente ao gênero Flavivirus, cuja transmissão ocorre através da picada do mosquito Aedes aegypti (VARELLA; JARDIM, 2009). A doença se tornou um grave problema de saúde pública, não só brasileiro como mundial, sendo considerada em expansão pela Organização Mundial da Saúde (OMS) (GABRIEL et al., 2018). Logo, essa doença infecciosa é uma das mais relevantes na maioria dos países tropicais e subtropicais (MONTEIRO; ARAÚJO, 2020), uma vez que as condições ambientais (temperatura, precipitação pluviométrica, processo de urbanização, etc.) favorecem a presença de criadouros, que permitem $o$ desenvolvimento e a proliferação do mosquito Aedes Aegypti, o vetor da doença (ARAQUAN, 2014).

Segundo a Organização Pan-Americana da Saúde (OPAS), os países e territórios do continente americano notificaram mais de 3 milhões de casos de dengue em 2019, o maior número registrado na região até o momento (OPAS, 2020). Somente no Brasil, ocorreram mais de 2 milhões de casos no referido ano, correspondente a $70 \%$ do total registrado e com mais da metade das mortes ocasionadas pela doença (OPAS, 2020).

De acordo com Gabriel et al. (2018), tratase de uma doença infecciosa aguda febril, que apresenta quatro tipos de vírus, sendo eles sorotipos: DENV-1, DENV-2, DENV-3 e DENV-4, as quais se diferenciam pela forma e intensidade como se manifestam. Por isso, encontra-se dificuldade em diagnosticar a doença, confundindo-a com outras que causam febre. A infecção por um dos sorotipos só confere imunidade permanente, ou no mínimo duradoura, para aquele sorotipo (TAUIL, 2001).

Segundo Varella e Jardim (2009), estudos recentes conjecturam que o vírus da dengue tenha surgido há 2 mil anos na África. Portanto, no século $\mathrm{XVI}$, teria circulado diversas vezes nas rotas do continente africano para o americano dentro dos navios, decorrente da popular mão de obra escrava da época, em um ambiente favorável para a sua proliferação, tais como em barris de água potável e em poças d'água.

Na América, é provável que os primeiros casos tenham surgido no século XVII. Entretanto, ainda são apenas especulações, já que os sintomas da dengue podem ser confundidos com os de outras enfermidades, principalmente da febre amarela, que é causada pelo mesmo transmissor, e também devastou a América nesse mesmo período (VARELLA; JARDIM, 2009).

Já em relação ao Brasil, no estado de São Paulo existem registros de epidemia de dengue desde 1850 (VARELLA; JARDIM, 2009). Dentre os anos de 1920 a 1980, não houve relatos de infectados pelo vírus, sendo considerada uma doença erradicada nacionalmente e em outros 17 países do continente americano entre 1950 a 1960. Porém, a infestação voltou entre os anos de 1981 e 1982, em Boa Vista-RO, e depois surgiu em 1986 no Rio de Janeiro-RJ, causada pelo vírus de sorotipo 1 (CLARO; TOMASSINO; ROSA, 2004). Já em 1987, houve o primeiro surto de dengue nos munícipios de Guararapes e Araçatuba, no estado de São Paulo (GABRIEL et al., 2018).

Entre os anos de 1990 e 1991, o sorotipo 2 causou uma nova epidemia, na qual ocorreram 1.952 casos de dengue hemorrágica com 24 mortes. Já no término dos anos 2000 , registrouse o isolamento do sorotipo 3 no estado do Rio de Janeiro, tido como o mais agressivo dentre os outros tipos de vírus (CLARO; TOMASSINO; ROSA, 2004).

A alta densidade populacional, advinda da urbanização, é um fator fundamental para a proliferação do mosquito Aedes aegypti, devido ao saneamento básico e moradias precárias (GABRIEL et al., 2018). Outro fator implicante na proliferação do mosquito seria o sistema produtivo industrial moderno, no qual a intensa produção de recipientes descartáveis, como 
plásticos e latas, contribui para o aumento do mosquito, principalmente, quando atirados em quintais, terrenos baldios ou/e em vias públicas, sendo locais inadequados para o descarte (TAUIL, 2001).

Gubler (1997) destaca que a dengue é uma doença influenciada por variáveis como temperatura, umidade relativa do ar e precipitação, sendo a pluviosidade um fator importante para o surgimento de potenciais criadouros, para a sobrevivência e reprodução do mosquito. Dessa forma, a incidência da dengue pode ser agravada devido às consequências das mudanças climáticas.

Tauil (2001) ressalta a importância da incorporação de hábitos populacionais de maneira cotidiana, colocando em prática ações como evitar reservatórios de água em quintais, trocar periodicamente a água das plantas e manter piscinas com água tratada. Deve-se levar em conta ainda que as ações de prevenção da dengue necessitam do envolvimento de outros setores da sociedade, com foco na melhoria das condições de urbanização e de habitação, na coleta regular de lixo, no abastecimento permanente de água encanada e na educação escolar.

Gabriel et al. (2018) destacam ainda que tais ações de prevenção e controle de dengue não são contínuas, sendo retomadas e reforçadas apenas no verão. Portanto, o Governo Federal precisa intensificar estratégias, tais como, campanhas publicitárias e educativas, mutirões de faxina, além de vigilância epidemiológica, em uma ação satisfatória que envolva todos os determinantes da dengue, controlando os transmissores do mosquito.

$\mathrm{O}$ aumento acentuado do número de casos de dengue é resultado de vários fatores, que facilitam a introdução, a transmissão e a dispersão do vírus, tais como: a utilização de meios de transportes cada vez mais rápidos; o aumento do processo de migração humana; a urbanização desordenada; a fragilidade da estrutura e planejamento, principalmente das periferias; o acréscimo do uso de recipientes descartáveis e a falta de saneamento ambiental (SCANDAR, 1998).

Diante do exposto, é importante que sejam desenvolvidos estudos e/ou ferramentas metodológicas, como forma de municiar a administração pública à elaboração de políticas públicas voltadas para a prevenção, monitoramento, controle e erradicação da doença e de seu vetor ao longo dos anos. Nesse sentido, estudos que contribuam para o conhecimento sobre o comportamento ou o movimento de índices de incidência de dengue em uma determinada região, de forma a acompanhar a sua dinâmica de transmissão e fazer previsões, são essenciais para auxiliar os gestores, evitando que se torne uma epidemia, e afete substancialmente a saúde pública e a qualidade de vida da população.

Assim, o objetivo deste trabalho é analisar a incidência de dengue em municípios da região da Alta Paulista. Especificamente, foi feito o ajuste de modelos fixos de séries temporais às séries históricas de incidência de dengue nos seis principais municípios da referida região, bem como previsões de incidência, usando as modelagens que se ajustaram melhor aos dados.

\section{MATERIAL E MÉTODOS}

Trata-se de uma pesquisa descritiva quanto aos objetivos com abordagem quantitativa, cuja unidade de análise são índices mensais de incidência de dengue.

Para a realização deste trabalho foram consideradas séries temporais de índices mensais de dengue referentes ao período de janeiro de 2010 a janeiro de 2020 (totalizando 121 observações cada uma) para os seis principais municípios que compõem a região da Alta Paulista: Adamantina, Dracena, Garça, Marília, Osvaldo Cruz e Tupã.

Os dados foram analisados primeiramente por meio de técnicas de análise baseadas em estatística descritiva (gráficos, tabelas e medidas descritivas) e, em seguida, em modelagem de séries temporais.

Neste trabalho foram consideradas técnicas de análise de séries temporais de modelo fixo, as quais são de fácil implementação e utilização, pois apresentam equações definidas baseadas em avaliações a priori da existência de determinadas componentes nas séries históricas, e se ajustam rapidamente às mudanças no comportamento de tais séries. Essas técnicas não exigem o uso de séries muito longas e são apropriadas para previsões de curto ou médio prazo. Dentre as técnicas de séries temporais de modelo fixo existentes (WANKE; JULIANELLI, 2006; OLIVEIRA et al., 2012), foram consideradas as que seguem:
a) Média Simples (MS)
b) Média Móvel Simples (MMS)
c) Alisamento Exponencial Simples (AES) 
d) Alisamento Exponencial Duplo (AED Método de Holt)

e) Alisamento Exponencial Triplo (AET Método de Winter aditivo)

Tais técnicas estão descritas na Tabela 1 , em que $P_{t+p}$ : previsão para os próximos $p$ períodos futuros (quando $p=1$, tem-se a previsão para o próximo período); $R_{t}:$ valor real observado no período $t ; n$ : número de informações da série; $M_{t}$ : média móvel no período $t$; $k$ : número de períodos considerados na média móvel; $\alpha$ : coeficiente de amortecimento que extrai da série a aleatoriedade; $\beta$ : coeficiente de amortecimento para a estimativa da tendência; $\gamma$ : coeficiente de amortecimento para a estimativa da sazonalidade; $N_{t}$ : componente nível; $T_{t}$ : componente tendência; e $S_{t}$ : componente sazonal.

Tabela 1. Técnicas de Modelos Fixos.

\begin{tabular}{|c|c|}
\hline $\begin{array}{c}\text { (a) Média } \\
\text { Simples (MS) }\end{array}$ & $P_{t+1}=\sum_{t=1}^{n} R_{t} / n$ \\
\hline $\begin{array}{l}\text { (b) Média } \\
\text { Móvel Simples } \\
\text { (MMS) }\end{array}$ & $P_{t+1}=\left(R_{t}+R_{t-1}+R_{t-2}+\ldots+R_{t-k+1}\right) / k$ \\
\hline $\begin{array}{l}\text { (c) Alisamento } \\
\text { Exponencial } \\
\text { Simples (AES) } \\
\end{array}$ & $P_{t+1}=\alpha R_{t}+(1-\alpha) P_{t}$ \\
\hline $\begin{array}{c}\text { (d) Alisamento } \\
\text { Exponencial } \\
\text { Duplo (AED) - } \\
\text { Holt }\end{array}$ & $\begin{array}{c}N_{t}=\alpha R_{t}+(1-\alpha)\left(N_{t-1}+T_{t-1}\right) \\
T_{t}=\beta\left(N_{t}-N_{t-1}\right)+(1-\beta) T_{t-1} \\
P_{t+p}=N_{t}+p T_{t}\end{array}$ \\
\hline $\begin{array}{l}\text { (e) Alisamento } \\
\text { Exponencial } \\
\text { Triplo (AET) - } \\
\text { Winters aditivo }\end{array}$ & $\begin{array}{c}S_{t}=\gamma\left(R_{t} / N_{t}\right)+(1-\gamma) S_{t-c} \\
T_{t}=\beta\left(N_{t}-N_{t-1}\right)+(1-\beta) T_{t-1} \\
N_{t}=\alpha\left(R_{t} / S_{t-c}\right)+(1-\alpha)\left(N_{t-1}+T_{t-1}\right) \\
P_{t+p}=\left(N_{g}+p T_{t}\right) S_{t-c+p}\end{array}$ \\
\hline
\end{tabular}

Fonte: Adaptado de Oliveira et al. (2012).

A seleção da técnica de análise de séries temporais mais adequada para a previsão do índice de incidência de dengue foi feita por meio da avaliação da precisão obtida com a aplicação de cada técnica relacionada anteriormente, (a)(e), optando-se pela técnica que apresentou o menor erro residual (diferença entre os valores reais e os valores previstos). Para definir a precisão dos modelos de séries temporais considerados, foi utilizado o Desvio Absoluto Médio (MAD) e o Erro Quadrático Médio (MSE), descritos a seguir, uma vez que as séries de incidência de dengue envolvem valores nulos, implicando em divisões por zero e inviabilizando o uso de outros métodos (WANKE; JULIANELLI, 2006; OLIVEIRA; ANDRADE, 2012):

$$
\begin{aligned}
& \mathrm{MAD}=\sum_{t=1}^{n}\left|R_{t}-P_{t}\right| / n \\
& \mathrm{MSE}=\sum_{t=1}^{n}\left(R_{t}-P_{t}\right)^{2} / n
\end{aligned}
$$

em que $R_{t}$ : valor real observado no período $t ; P_{t}$ : previsão para o período $t$ e $n$ : número de informações da série.

Assim, tais métodos foram usados como ferramentas para definir a técnica mais robusta na previsão do índice de incidência de dengue e, posteriormente, usando a técnica escolhida, foram obtidas duas previsões: uma dentro do período analisado (Janeiro de 2020) e a outra fora do período analisado (Fevereiro de 2020).

A precisão obtida pelos métodos de Alisamento Exponencial está associada à seleção dos coeficientes de amortecimento $\alpha$ (componente de aleatoriedade), $\beta$ (componente de tendência) e $\gamma$ (componente de sazonalidade), ou seja, escolhem-se valores que minimizem o erro da previsão (WANKE; JULIANELLI, 2006; OLIVEIRA et al., 2012).

Neste trabalho, a estatística descritiva, a seleção dos coeficientes e o ajuste dos modelos foram realizados pelo software Minitab. 


\section{RESULTADOS E DISCUSSÃO}

3.1. Análise descritiva dos índices de incidência de dengue

A Tabela 2 mostra as principais medidas descritivas relacionadas ao número de casos de dengue nos municípios da região da Alta Paulista no período analisado, evidenciando a grande variabilidade (elevado desvio-padrão) de um ano para outro da incidência da doença na região.

Tabela 2. Medidas descritivas referentes ao número de casos de dengue nos munícipios da região da Alta Paulista - Jan/2010 a Jan/2020.

\begin{tabular}{l|r|r|c|r}
\hline Município & Média & $\begin{array}{r}\text { Desvio } \\
\text { padrão }\end{array}$ & Mínimo & Máximo \\
\hline Adamantina & 33,72 & 108,49 & 0 & 829 \\
\hline Dracena & 21,64 & 55,88 & 0 & 350 \\
\hline Garça & 21,06 & 74,99 & 0 & 572 \\
\hline Marília & 139,05 & 483,43 & 0 & 3376 \\
\hline Osv. Cruz & 35,93 & 102,42 & 0 & 679 \\
\hline Tupã & 113,23 & 347,66 & 0 & 2470 \\
\hline
\end{tabular}

Fonte: Secretaria de Estado da Saúde (2020).

A dispersão mostrada na Tabela 2 é confirmada pela Figura 1, que possibilita uma visão geral do comportamento dos índices mensais de incidência da doença no período.

Figura 1. Evolução do número de casos de dengue na região da Alta Paulista - Jan/2010 a Jan/2020.

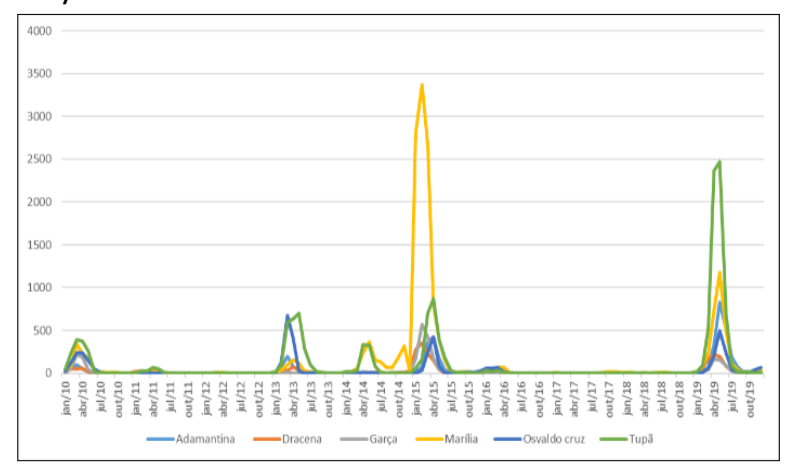

Fonte: Secretaria de Estado da Saúde (2020).

Observa-se ainda que ocorreram picos de casos de dengue nos anos de 2010, 2013, 2015 e 2019 em todos os municípios estudados, sendo mais evidenciados no período entre janeiro e julho de cada ano. Apenas os municípios de Marília e de Tupã apresentaram casos no ano de 2014.

3.2. Ajuste dos modelos fixos às séries de índice de incidência de dengue

As Tabelas 3 a 8 mostram os modelos ajustados às séries de incidência de dengue nos seis municípios da região da Alta Paulista considerados neste trabalho, bem como os valores obtidos para cada técnica de seleção de modelos (MAD e MSE), o que permite verificar qual deles teve o melhor ajustamento para os dados do período analisado.

De acordo com os resultados das Tabelas 3 a 8, averiguou-se que quatro municípios (Dracena, Garça, Marília e Osvaldo Cruz) apresentaram menores erros (ou mais precisão) para o modelo Alisamento Exponencial Simples (AES) nas comparações feitas por meio dos métodos MAD e MSE.

Tabela 3. Modelos ajustados à série de incidência de dengue do município de Adamantina.

\begin{tabular}{crr}
\hline Método de Previsão & MAD & MSE \\
\hline MS & 42,46 & 12062,8 \\
MMS (ordem dois) & 34,94 & 9839,37 \\
\hline $\begin{array}{c}\text { AES } \\
\alpha=1,548\end{array}$ & 13,17 & 1648,16 \\
\hline $\begin{array}{c}\text { AED-Holt } \\
\alpha=1,533 ; b=0,003\end{array}$ & $\mathbf{1 3 , 1 1}$ & $\mathbf{1 5 6 5 , 8 9}$ \\
\hline $\begin{array}{c}\text { AET-Winter } \\
\alpha=1,000 ; 6=0,003 ; \gamma=0,050\end{array}$ & 31,80 & 6531,68 \\
\hline
\end{tabular}


Tabela 4. Modelos ajustados à série de incidência de dengue do município de Dracena.

\begin{tabular}{crc}
\hline Método de Previsão & MAD & MSE \\
\hline MS & 29,96 & 3833,80 \\
\hline MMS (ordem dois) & 20,46 & 2691,53 \\
\hline $\begin{array}{c}\text { AES } \\
\alpha=1,499\end{array}$ & $\mathbf{7 , 3 1}$ & $\mathbf{4 1 2 , 5 3}$ \\
\hline $\begin{array}{c}\text { AED-Holt } \\
\alpha=1,529 ; \boldsymbol{B}=0,005\end{array}$ & 8,06 & 465,93 \\
\hline $\begin{array}{c}\text { AET-Winter } \\
\alpha=1,000 ; \boldsymbol{0}=0,005 ; \gamma= \\
0,050\end{array}$ & 18,36 & 1905,34 \\
\hline
\end{tabular}

Tabela 5. Modelos ajustados à série de incidência de dengue do município de Garça.

\begin{tabular}{crc}
\hline Método de Previsão & MAD & MSE \\
\hline MS & 37,90 & 5932,93 \\
\hline MMS (ordem dois) & 22,23 & 4853,80 \\
\hline AES & $\mathbf{8 , 7 4}$ & $\mathbf{7 5 4 , 4 9}$ \\
$\alpha=1,538$ & 8,75 & 756,16 \\
\hline $\begin{array}{c}\text { AED-Holt } \\
\alpha=1,216 ; \boldsymbol{b}=0,010\end{array}$ & 18,01 & 3189,27 \\
$\begin{array}{c}\text { AET-Winter } \\
\alpha=1,000 ; \beta=0,010 ; \gamma= \\
0,050\end{array}$ & & \\
\hline
\end{tabular}

Apenas os municípios de Adamantina e Tupã obtiveram melhores ajustes usando o modelo Alisamento Exponencial Duplo (AED). Esse fato pode ser explicado possivelmente porque os municípios de Adamantina e Tupã apresentaram curvas bastante similares de transmissão (vide Figura 1).

Tabela 6. Modelos ajustados à série de incidência de dengue do município de Marília.

\begin{tabular}{ccc}
\hline Método de Previsão & MAD & MSE \\
\hline MS & 190,12 & 23750,51 \\
\hline MMS (ordem dois) & 130,46 & 16958,09 \\
\hline $\begin{array}{c}\text { AES } \\
\alpha=1,249\end{array}$ & $\mathbf{2 1 , 7 1}$ & $\mathbf{6 8 0 5 , 0 5}$ \\
\hline $\begin{array}{c}\text { AED-Holt } \\
\alpha=1,368 ; 6=0,010\end{array}$ & 33,58 & 1522,91 \\
\hline $\begin{array}{c}\text { AET-Winter } \\
\alpha=1,000 \text { B }=0,010 ; \gamma \\
=0,050\end{array}$ & 120,53 & 11433,31 \\
\hline
\end{tabular}

Tabela 7. Modelos ajustados à série de incidência de dengue do município de Osvaldo Cruz.

\begin{tabular}{ccc}
\hline Método de Previsão & MAD & MSE \\
\hline MS & 61,29 & 11583,69 \\
\hline MMS (ordem dois) & 41,16 & 11926,91 \\
\hline $\begin{array}{c}\text { AES } \\
\alpha=1,306\end{array}$ & $\mathbf{9 , 8 8}$ & $\mathbf{8 1 6 , 2 3}$ \\
\hline $\begin{array}{c}\text { AED-Holt } \\
\alpha=1,372 ; \boldsymbol{\theta}=0,008\end{array}$ & 13,24 & 1219,80 \\
\hline $\begin{array}{c}\text { AET-Winter } \\
\alpha=1,000 ; \boldsymbol{B}=0,008 ; \\
0,050\end{array}$ & 39,18 & 8936,68 \\
\hline
\end{tabular}

Tabela 8. Modelos ajustados à série de incidência de dengue do município de Tupã.

\begin{tabular}{crr}
\hline Método de Previsão & MAD & \multicolumn{1}{c}{ MSE } \\
\hline MS & 154,63 & $\begin{array}{r}12241,2 \\
3\end{array}$ \\
\hline MMS (ordem dois) & 117,12 & $\begin{array}{r}10934,6 \\
8\end{array}$ \\
\hline $\begin{array}{c}\text { AES } \\
\alpha=1,684\end{array}$ & 61,64 & 2518,72 \\
\hline $\begin{array}{c}\text { AED-Holt } \\
\alpha=1,674 ; 6=0,010\end{array}$ & 60,82 & $\mathbf{2 4 7 9 , 6 5}$ \\
\hline $\begin{array}{c}\text { AET-Winter } \\
\alpha=1,000 ; 0=0,010 ; \\
0,050\end{array}$ & 82,25 & 7128,85 \\
\hline
\end{tabular}

Com relação aos métodos de Média Simples (MS) e Média Móvel Simples (MMS), contatou-se que estes não foram significativos para nenhum caso, pois não acompanharam os movimentos de incidência de dengue no período analisado. Nesses casos, na maioria das vezes as previsões de média simples e de média móvel simples superestimaram os verdadeiros valores dos índices de dengue.

\subsection{Previsão de incidência de dengue com base nos melhores modelos ajustados}

Baseando-se nos melhores modelos ajustados, foi feita uma previsão de incidência de dengue para os meses de janeiro e de fevereiro de 2020, conforme Tabela 9 a seguir. 
Tabela 9. Previsão de casos de dengue para Jan/2020 e Fev/2020, conforme o melhor modelo ajustado para cada município.

\begin{tabular}{c|c|r|r}
\hline Município & $\begin{array}{c}\text { Melhor } \\
\text { modelo } \\
\text { ajustado }\end{array}$ & $\begin{array}{c}\text { Previsão } \\
\text { de casos } \\
\text { para } \\
\text { Jan/2020 } \\
\text { Fev/2020 }\end{array}$ & $\begin{array}{c}\text { Número } \\
\text { real de } \\
\text { casos em } \\
\text { Jan/2020 } \\
\text { Fev/202 } \\
0\end{array}$ \\
\hline Adamantina & AED-Holt & 238 & 165 \\
Dracena & AES & 245 & 311 \\
\hline Garça & AES & 429 & 302 \\
\hline Marília & AES & 158 & 593 \\
\hline Osv. Cruz & AES & 224 & 114 \\
& & 409 & 195 \\
\hline Tupã & AED-Holt & 818 & 221 \\
\hline T. & & 95 & 289 \\
\hline
\end{tabular}

Fonte: Governo do Estado de São Paulo. Secretaria de Estado da Saúde (2021).

Os resultados apresentados mostraram que os modelos de alisamento exponencial são os mais robustos para ajustar os dados de incidência de dengue e, consequentemente, para realizar previsões.

Pindyck e Rubinfeld (2004) afirmam que o processo de suavização (ou alisamento) é bastante útil quando se deseja remover ou pelo menos reduzir flutuações de curto-prazo de uma série temporal. Isso significa que métodos de alisamento exponencial são indicados quando se tem o objetivo de facilitar a interpretação e a análise dos dados.

Por outro lado, Makridakis, Wheelwright e Hyndman (1998) investigaram o poder preditivo de vários métodos comumente utilizados na previsão de séries temporais e constataram que o incremento da complexidade e da sofisticação estatística dos métodos de previsão de séries temporais não implica, necessariamente, em uma melhoria na acuidade da previsão. Eles relataram que "métodos simples de previsão podem apresentar desempenho extremamente satisfatório sob certas condições". Além disso, métodos de previsão menos complexos normalmente permitem alcançar total compreensão de suas suposições e limitações, e de interpretação de seus resultados (RIBEIRO et al., 2011; ANDRADE; OLIVEIRA, 2011; OLIVEIRA et al., 2012).

\section{CONSIDERAÇÕES FINAIS}

Estudos sobre incidência de dengue são fundamentais ao acompanhamento da dinâmica e à previsão de transmissão da doença, impactando e contribuindo para a elaboração de políticas públicas destinadas à saúde.

Nesse sentido, o presente trabalho apresenta $\mathrm{o}$ ajuste de diferentes modelos estatísticos que proporcionam justamente um acompanhamento e previsão de casos de dengue.

Observou-se que os modelos de alisamento exponencial foram os mais eficientes e eficazes ao ajustar e prever índices de incidência de dengue em municípios da região da Alta Paulista. Esses modelos apresentaram menores erros de estimação, proporcionando melhores previsões para tais índices.

Uma vez que as séries históricas de incidência de dengue possuem muitos valores nulos (excesso de zeros), os modelos fixos muitas vezes não conseguem acompanhar os movimentos das séries de forma eficaz. Logo, a aplicação de mais de um modelo de previsão e a verificação da sua consistência podem e devem ser consideradas, propiciando um aumento na confiabilidade da previsão. Assim, sugere-se como propostas futuras o ajuste de modelos abertos (Modelos Autorregressivos, Integrados e de Médias Móveis - ARIMA) ou ainda de modelos lineares generalizados com distribuição de Poisson e zeros inflados, como forma de obter melhores previsões.

\section{REFERÊNCIAS}

ANDRADE, M. G.; OLIVEIRA, S. C. A comparative study of Bayesian and maximum likelihood approaches for Arch models with evidence from Brazilian financial series. New Mathematics and Natural Computation, v. 7, p. 347-361, 2011. https://doi.org/10.1142/S1793005711001974

ARAQUAN, R. B. Análise da incidência de dengue nos distritos regionais de Belo Horizonte-MG, entre os anos de 2005 a 2013. 2014. Dissertação (Mestrado em Geografia) - Universidade Federal de Viçosa, UFV, Viçosa, MG, 2014.

CLARO, L. B. L.; TOMASSINI, H. C. B.; ROSA, M. L. G. Prevenção e controle do dengue: uma revisão de estudos sobre conhecimentos, crenças e 
práticas da população. Cad. Saúde Pública, Rio de Janeiro, v. 20, n. 6, p. 1447-1457, Dec. 2004. https://doi.org/10.1590/S0102-

\section{X2004000600002}

GABRIEL, A. F. B.; ABE, K. C.; GUIMARAES, M. de P.; MIRAGLIA, S. G. El K. Avaliação de impacto à saúde da incidência de dengue associada à pluviosidade no município de Ribeirão Preto, São Paulo. Cad. saúde colet., Rio de Janeiro, v. 26, n. 4 p. 446-452, Dec. 2018. https://doi.org/10.1590/1414$\underline{462 \times 201800040119}$

GOVERNO DO ESTADO DE PAULO. Secretaria de Estado da Saúde. Dados Estatísticos. São Paulo: Secretaria de Estado de Saude, 2021. Disponível em: http://saude.sp.gov.br/cve-centro-devigilancia-epidemiologica-prof.-alexandrevranjac/oldzoonoses/dengue/dados-estatisticos\#. Acesso em: 02 jun. 2020.

GUBLER, D. J. Dengue and dengue hemoragic fever: its history and resurgence as a global health problem. In: GUBLER, D. J.; KUNO, G. (ed.) Dengue and dengue he morragic fever. New York: CAB International, 1997. p.1-22. https://doi.org/10.1079/9781845939649.0001

MAKRIDAKIS, S.; WHEELWRIGHT, S.; HYNDMAN, R. Forecasting: Methods and Applications. New York: John Wiley \& Sons, 1998.

MONTEIRO, V. B.; ARAÚJO, J. A. Aspectos socioeconômicos e climáticos que impactam a ocorrência de dengue no Brasil: análise municipal de 2008 a 2011 por regressões quantílicas para dados em painel. Revista Brazilian Journal of Development, Curitiba, v. 6, n. 5, p. 2812628145, maio. 2020. https://doi.org/10.34117/bjdv6n5-311

OLIVEIRA, S. C.; PEREIRA, L. M. M.; HANASHIRO, J. T. S.; VAL, P. C. A study about the performance of time series models for the analysis of agricultural prices. GEPROS. Gestão da Produção, Operações e Sistemas, v. 3, p. 11-27, 2012. https://doi.org/10.15675/gepros.v7i3.770

OLIVEIRA, S. C.; ANDRADE, M. G. Comparison between the complete Bayesian method and empirical Bayesian method for ARCH models using Brazilian financial time series. Pesquisa Operacional, v. 32, n. 2, p. 293-313, 2012.
http://dx.doi.org/10.1590/S0101$\underline{74382012005000019}$.

OPAS. ORGANIZAÇÃO PAN-AMERICANA DA SAÚDE. Casos de dengue nas Américas ultrapassam 3 milhões em 2019. Brasília: OPAS, $2019 . \quad$ Disponível em: https://www.paho.org/bra/index.php?option=co m_content\&view=article\&id=6108: casos - dedengue-nas-americas-ultrapassam-3-milhoes-em2019\&Itemid=812. Acesso em: 07 jun. 2020.

PINDYCK, R.; RUBINFELD, D. Econometria: Modelos e Previsões. Rio de Janeiro: Elsevier, 2004.

RIBEIRO, S. G. B.; OLIVEIRA, S. C.; SCALCO, A. R.; PINTO, L. B. O comportamento do preço do leite tipo $C$ pago ao produtor no estado de São Paulo confrontado com a realidade dos produtores da microrregião de Marília. Revista Brasileira de Engenharia de Biossistemas, v. 5, p. 117-127, 2011.

http://dx.doi.org/10.18011/bioeng2011v5n2p117 $\underline{-127}$

SCANDAR, S. A S. Dengue no município de Paraíso. São Paulo/Brasil 1993. 1998. Dissertação (Mestrado em Epidemiologia) - Universidade Federal de São Paulo, UNIFESP, São Paulo, 1998.

TAUIL, P. L. Urbanização e ecologia do dengue. Cad. Saúde Pública, Rio de Janeiro, v. 17, (supl.). p. S99-S102, 2001.

https://doi.org/10.1590/S0102$\underline{311 \times 2001000700018 .}$.

VARELLA, D.; JARDIM, C. Coleção Doutor Drauzio Varella: Dengue e Febre Amarela. Barueri: Gold, 2009. 63p.

WANKE, P.; JULIANELLI, L. (orgs.) Previsão de vendas: Processos organizacionais e métodos quantitativos e qualitativos. São Paulo: Atlas, 2006. 